\section{$\underset{\substack{\text { hommes } \\ \text { \& migrations }}}{ }$}

\section{Hommes \& migrations}

Revue française de référence sur les dynamiques

migratoires

$1293 \mid 2011$

L'immigration dans les musées

\title{
Les institutions culturelles face aux histoires et aux mémoires d'immigration
}

Vingt-cinq ans d'expériences en Rhône-Alpes

\section{Benjamin Vanderlick}

\section{(2) OpenEdition}

Journals

\section{Édition électronique}

URL : http://journals.openedition.org/hommesmigrations/513

DOI : 10.4000/hommesmigrations.513

ISSN : 2262-3353

Éditeur

Musée national de l'histoire de l'immigration

\section{Édition imprimée}

Date de publication : 1 septembre 2011

Pagination : 86-95

ISSN : $1142-852 X$

\section{Référence électronique}

Benjamin Vanderlick, «Les institutions culturelles face aux histoires et aux mémoires d'immigration », Hommes \& migrations [En ligne], 1293 | 2011, mis en ligne le 31 décembre 2013, consulté le 01 mai 2019. URL : http://journals.openedition.org/hommesmigrations/513; DOI : 10.4000/

hommesmigrations.513 


\section{Les institutions culturelles face aux histoires et aux mémoires d’immigration}

Vingt-cinq ans d'expériences en Rhône-Alpes

Par Benjamin Vanderlick, Ethnologue - photographe

L'immigration, dans la mesure où elle est associée à un territoire, constitue une source vive pour les projets muséographiques régionaux.

Pendant une dizaine d'années, en région Rhône-Alpes, les projets

d'expositions qui offrent une place à l'expression des mémoires de l'immigration se multiplient. De l'angle historique à l'approche sociale ou culturelle, les manières de traiter ce thème varient considérablement.

Ces différences montrent que, loin de recevoir une réponse univoque, la question de l'immigration suscite un intérêt croissant. 
La région Rhône-Alpes a vu naître une multitude d'expériences artistiques, expositions, installations, événements culturels, films, spectacles qui permettent de valoriser les histoires et les mémoires d'immigration. Pendant dix ans, de 2000 à 2009, le projet "Traces en Rhône-Alpes, forum régional des mémoires d'immigrés ${ }^{(1)}$ " a notamment permis de fédérer une centaine de partenaires aussi bien institutionnels qu'associatifs et ainsi de créer et réunir une diversité de projets culturels et artistiques sur l'ensemble des départements de la région : films, pièces de théâtre, expositions. Les Journées européennes du patrimoine ont, depuis dix ans, elles aussi servi de prétexte à valoriser l'immigration dans l'histoire régionale : organisation de parcours dès 1999 par l'association Peuplement et migrations sur les traces de l'histoire de l'immigration régionale, mise en place d'un programme patrimonial en 2009 à Modane (Savoie), à la Guillotière (Lyon) en partenariat avec les municipalités concernées et la Cité nationale de l'histoire de l'immigration...

Ces initiatives, souvent menées avec le soutien de la politique de la ville, représentent la majorité de la production régionale sur cette thématique. Des projets culturels de grande qualité, soutenus par les politiques publiques, sont aussi réalisés. Initiés en grande majorité par des associations, ils se situent toutefois à l'écart des institutions culturelles régionales.

Ces dernières se sont pourtant engagées dans des démarches de prise en considération de l'immigration locale au sein de leur programmation, en accord avec l'esprit de leur lieu. Il s'agit d'interroger le traitement de l'immigration dans les musées de la région, son évolution depuis vingt-cinq ans, à travers l'évocation de la diversité des expériences muséographiques réalisées.

\section{Une expérience atypique ancienne: le Musée dauphinoîs à Grenoble (Isère)}

Dans la région Rhône-Alpes, une institution fait figure de pionnière en la matière : le Musée dauphinois. Musée municipal jusqu'en 1992 avant de devenir musée départemental, il s'inscrit dans le courant écomuséologique des années soixante et soixante-dix et souhaite se présenter comme un lieu d'interprétation de la ville et de son environnement alpin. Les projets menés par le musée s'orientent autour de cinq thématiques : la montagne, les modes de vie des montagnards hier et aujourd'hui; l'archéologie ; l'histoire et le patrimoine régionaux ; le patrimoine industriel ; les faits de société, les minorités. Le travail sur l'immigration prend place dans la dernière thématique. Le premier projet expographique dans lequel le musée traite de l'immigration date de 1982 et s'inscrit dans l'exposition Le Roman des Grenoblois, 
réalisée par Jean-Pierre Laurent. L'immigration algérienne est en effet évoquée à travers le récit fictif d'un père de famille maghrébin de la rue Très-Cloître. Certains Grenoblois ont manifesté leur désaccord face à cette figure du Grenoblois qui leur était proposée. Ils ont fait pression auprès de la municipalité pour demander la suppression de cet épisode, sans succès.

Par la suite, entre 1988 et 2010, se sontsuccédé une série de projets fondés sur l'origine géographique. En 1988, le jumelage de Grenoble avec la ville de Corato, dans les Pouilles, "une ville du sud de l'Italie qui compterait, dit-on, plus d'habitants à Grenoble qu'au lieu dit ${ }^{2}$ ", est l'élément déclencheur. Le Musée dauphinois réalise, avec la participation de représentants de cette communauté italienne, une première exposition sur les populations immigrées de la ville : Corato-Grenoble (1988). En s'appuyant sur une collecte de témoignages et d'objets, le projet présente les raisons de l'exil, l'arrivée à Grenoble, la transmission du patrimoine immatériel (dialecte, gastronomie...) et les réactions des Coratins au contact de la ville.

Un deuxième projet naît d'une demande de Grenoblois d'origine grecque en vue de présenter une exposition de costumes traditionnels de leur pays d'origine au Musée dauphinois. Avec ces personnes, la question de l'exode des Grecs d'Asie Mineure au début du $\mathrm{XX}^{\mathrm{e}}$ siècle refait surface et permet d'apporter des éléments de compréhension. Le musée s'appuie sur des parcours de vie pour réaliser l'exposition Des Grecs en 1993. Des protestations émanent du consulat de Turquie à Lyon qui voit dans ce projet une incitation à la détérioration des relations diplomatiques entre la Grèce et la Turquie. Cette expérience sert de déclencheur pour les descendants d'Arméniens qui ont survécu au génocide de 1915 et qui ont été nombreux à trouver du travail dans la vallée du Rhône et ses alentours. Cela aboutit, selon une démarche similaire, à la réalisation de l'exposition D'Isère et d'Arménie en 1997. Ces projets ont montré que la mise en valeur des témoignages concernant des phénomènes migratoires, alors que les derniers témoins avaient presque tous disparu, pouvait susciter de fortes émotions.

La projection au Musée dauphinois, par l'association Amitié et liens France-Maghreb $(\mathrm{ALIF})^{(3)}$, du film Mémoires d'immigrés (1997) de Yamina Benguigui est l'élément déclencheur d'un projet sur une immigration plus contemporaine et éminemment sensible : les Maghrébins, de leur arrivée en Isère après 1945, appelés pour développer les industries et les villes, au regroupement familial et enfin au déficit de reconnaissance et au racisme persistant hérités de la période coloniale. Jean-Claude Duclos, alors conservateur, a travaillé avec les associations locales, effectuant une collecte surtout orale, un peu iconographique et très peu matérielle. C'est l'exposition Pour que la vie continue. D'Isère et du Maghreb (1999). Les réactions sont nombreuses : réactions de soutien mais aussi de protestation, quelquefois violentes. L'exposition 
est prolongée d'un an. Les pieds-noirs, dont 35000 ont été rapatriés en Isère en 1962 et qui avaient vécu cette exposition comme un affront, ont été invités au musée à l'initiative d'élus. De ces discussions, et afin d'apaiser des plaies encore à vif, naîtra une autre exposition : Français d'Isère et d'Algérie (2003). Le Musée dauphinois a réalisé un autre projet, cette fois-ci avec les Isérois issus de toute l'Afrique, dans lequel plus d'une trentaine d'association sont impliquées : Ce que nous devons à l'Afrique (2010).

Dans un autre musée départemental d'Isère - le Musée de la résistance et de la dépor-

\section{La politique d'exposition initiée au Musée dauphinois et adoptée par le Musée de la résistance et de la déportation montre la possibilité d'évoquer l'immigration dans des expositions régionales.} tation/Maison des droits de l'homme -, dont la direction est également confiée à Jean-Claude Duclos, d'autres projets d'expositions en lien avec les immigrations locales se mettent en place au cours des années deux mille. Ce lieu accueille une exposition photographique sur Sangatte : Le Hangar (2002). En lien avec des associations locales sont aussi réalisées une exposition sur le Cambodge, Face au génocide, du Cambodge à l'Isère (2008), et une autre sur l'immigration espagnole issue de la guerre d'Espagne, Le train s'est arrêté à Grenoble (2009).

La politique d'exposition initiée au Musée dauphinois et adoptée par le Musée de la résistance et de la déportation montre la possibilité d'évoquer l'immigration dans des expositions régionales. La politique culturelle de ces deux musées d'Isère insiste non seulement sur l'importance de privilégier, pour ce type de projets, une muséographie participante mais aussi sur le rôle social auquel doit répondre un musée de patrimoine et de société sur son territoire.

\section{Une diversité de musées sensibles à la question}

D'autres musées ont questionné l'immigration en région dès la fin des années quatrevingt. C'est le cas de l'Écomusée Nord-Dauphiné de Saint-Quentin-Fallavier (Isère) qui effectua une mission ethnologique sur le territoire industriel de Pont-de-Chéruy. Cette agglomération de 35000 habitants du Nord-Isère, qui doit son développement économique à l'essor des entreprises de tréfileries Grammont, a accueilli, jusqu'à la crise industrielle des années soixante-dix et quatre-vingt, une diversité de vagues migratoires : Italiens dès la fin du XIX ${ }^{\mathrm{e}}$ siècle, puis Grecs, Arméniens, Russes, 
Espagnols, Portugais, Yougoslaves, Maghrébins, réfugiés d'Asie du Sud-Est, Turcs. L'Écomusée souligne, au travers de l'histoire industrielle, l'importance de l'immigration lors de son exposition Champs et sirènes (1989), avec le film Boulevard des Tréfileries (1989) et l'ouvrage Instants d'années (1990) qui valorise cette histoire industrielle singulière et ces histoires d'immigration. Dans les années quatre-vingtdix, l'ancien conservateur de cet écomusée, l'anthropologue Daniel Pelligra, devient directeur de l'association Peuplement et migrations et défend l'idée d'une reconnaissance des lieux de mémoire de l'immigration en Rhône-Alpes ${ }^{(4)}$ avec le souhait de créer un musée, L'Escale, cité du voyage et des échanges ${ }^{(5)}$,à Vaulx-en-Velin, dans l'ancienne usine TASE $^{(6)}$.

C'est surtout depuis dix ans que l'on assiste à une multiplication de projets d'expositions dans les musées qui considèrent l'immigration comme une donnée constituante de la société et de l'histoire. Le nombre d'institutions qui ont déjà évoqué cette thématique dans leur programmation reste encore faible au regard du nombre de musées et d'institutions culturelles de la région. Cette prise en considération de l'immigration comme sujet culturel et non social reste encore marginale dans les lieux institutionnels de la culture, en région comme en France.

Ces expériences, qui peuvent être considérées comme insuffisantes, nous permettent toutefois de rendre compte des différentes manières d'interroger ce phénomène sous forme muséographique dans les musées de la région Rhône-Alpes.

Ainsi, en 2003, la bibliothèque municipale de Lyon Part-Dieu confie à l'historien Philippe Videlier le soin de réaliser une exposition d'envergure, L'Algérie à Lyon, une mémoire centenaire. Ce projet, réalisé dans le cadre de l'Année de l'Algérie en France, est rendu possible grâce au concours de la délégation à l'intégration et aux droits des citoyens de la ville de Lyon. Par la suite, dans le cadre du forum Traces, le service de la documentation régionale de la bibliothèque réalise des expositions issues de ses fonds d'archives photographiques: Traces de migration, Marcelle Vallet (2005) puis Mémoire d'exils, photographies 1940-1970(2008)

Entre 2005 et 2010, on assiste au sein des musées de société à la réalisation de quelques projets d'expositions sur la région qui évoquent l'immigration. L'une des plus conséquentes est l'exposition Frontières, images de vies entre les lignes (2006-2007) présentée au Muséum de Lyon (appelé à devenir Musée des confluences) sur environ $700 \mathrm{~m}^{2}$. Cette exposition de géopolitique mondiale sur la thématique de la frontière proposa une réflexion sur le concept de frontière en s'appuyant sur plusieurs travaux photographiques accompagnés de textes de spécialistes pour chaque exemple traité. S'il est question de reportages sur les frontières États-Unis-Mexique, Israël-Palestine, indo-pakistanaise au Cachemire..., le musée intègre des expériences migratoires : le voyage clandestin de Kingsley suivi par le photographe Olivier Jobard, mais aussi les 
témoignages de demandeurs d'asile ou des photographies de Roms en bidonvilles dans l'agglomération lyonnaise. Cette démarche souhaitait alors inscrire une réalité locale au milieu d'un phénomène mondial.

À une échelle plus modeste, le musée départemental du Revermont (Ain) a pris en considération, dans une exposition sur les musiques populaires du territoire, la place des musiques des immigrations marocaine et turque en réalisant une collecte musicale dans la vallée industrielle d'Oyonnax : À la croisée des musiques, un air de fêtes $^{(8)}(2009-2010)$.

\section{Un sujet en quête d'autonomie}

Toutefois, l'immigration s'affiche sensiblement davantage au sein des musées d'histoire que dans les musées de société. Par exemple, le Musée urbain Tony Garnier (Lyon) a proposé en 2007 une exposition sur les baraques de l'entre-deuxguerres du quartier Gerland à Lyon ${ }^{(9)}$. Ce projet d'exposition fut réalisé à partir d'un travail historique du laboratoire Mémoires urbaines et archives ouvrières de l'ENS Lettres venu s'installer à Lyon dans le quartier qui, soixantedix ans plus tôt, abritait ce bidonville. Le Musée urbain, en proposant cette exposition, réfléchissait en premier lieu à un modèle d'urbanisme. Le laboratoire de recherche de l'ENS, quant à lui, interroge l'histoire ouvrière.

Lalbum photographique de l'organisme de bienfaisance qui sert de base à l'exposition ne fait quasiment pas état de l'immigration européenne qui existe dans ces installations de fortune. L'immigration se révèle entre les lignes dans cette exposition, pour qui s'intéresse au sujet, à travers

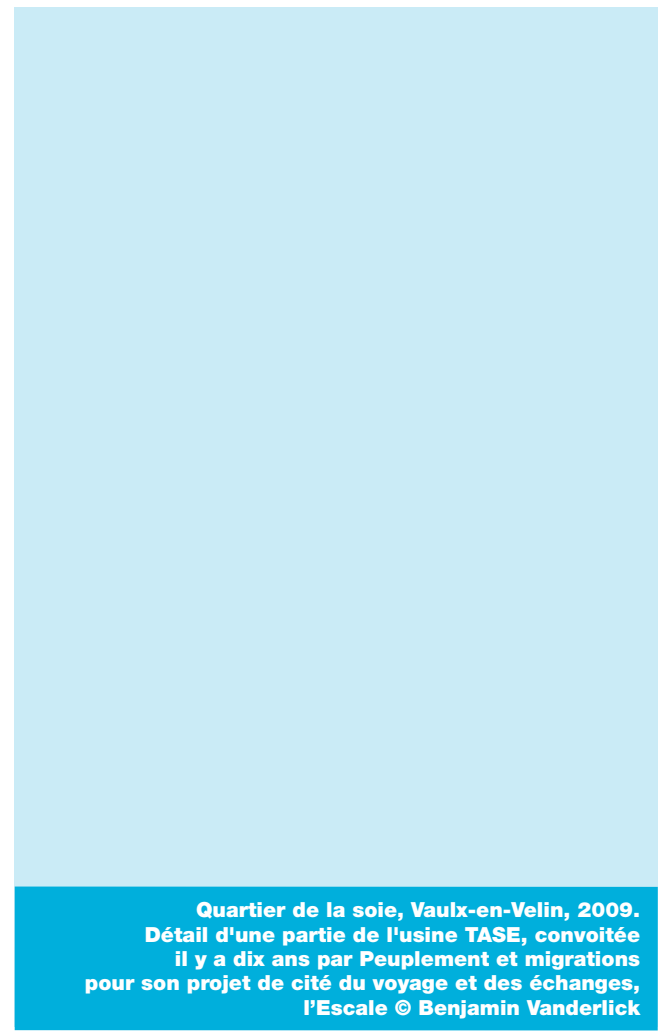


les textes des historiens mais surtout dans la contribution de l'historienne de l'immigration Marie-Claude Blanc-Chaléard ${ }^{(10)}$.

Selon une démarche relativement semblable, le Musée départemental de la viscose à Échirolles (Isère) a réalisé l'exposition Derrière l'usine, les cantonnements de Salaize-surSanne (décembre 2009-mai 2010). Ce projet est issu d'une étude sur les cantonnements et du souhait du laboratoire dirigeant le rapport de valoriser le produit de cette recherche ainsi que les photographies du comité d'entreprise des établissements Rhodia de Salaize-sur-Sanne (Isère). Les cantonnements, logeant exclusivement des travailleurs étrangers, constituent un support de mémoire aux témoignages d'ouvriers mais aussi d'immigrés.

Depuis quelques années, à Lyon, le Centre d'histoire de la résistance et de la déportation (CHRD) est conscient de la nécessité d'évoquer l'immigration tout en établissant des passerelles avec les origines de la création du lieu. Il s'agit en effet d'un musée de France municipal rattaché au service "Droits de l'homme" de la ville et non au service "Culture". Une exposition photo sur les tirailleurs africains a été programmée en 2007 : Anciens combattants africains. Des visages et des mots pour mémoire (de Philippe Guionie). En 2010, le CHRD présente sous forme d'exposition l'immigration

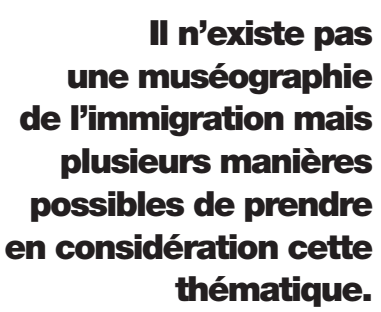
cette migration débute à la chute du mur de Berlin et à la fin du régime de Ceausescu, force est de constater que cette circulation, qui ne concerne qu'une infime partie des Roumains, est en permanente réadaptation. Ce projet trouve, par ailleurs, une pertinence en s'inscrivant dans le prolongement d'une exposition sur l'internement des Tsiganes durant la Seconde Guerre mondiale, en offrant un regard contemporain sur un peuple européen encore stigmatisé.

Ces exemples montrent que la volonté de questionner l'immigration dans ces musées demeure timide. Il reste à souligner que la manière d'exposer l'immigration dans les musées est extrêmement variée en Rhône-Alpes. Chacun va en effet aborder l'immigration selon son savoir-faire et sa technique muséographique. Il n'existe pas une muséographie de l'immigration mais plusieurs manières possibles de prendre en considération cette thématique. 


\section{La création de nouveaux lieux dédiés à l'immigration}

Au-delà des musées précédemment évoqués, les dynamiques mémorielles du $\mathrm{XXI}^{\mathrm{c}}$ siècle donnent naissance à de nouveaux lieux dédiés à l'histoire et aux mémoires, où l'immigration sera inscrite quasi naturellement dans les axes de travail.

À Valence (Drôme) est créé en 2005 le Centre du patrimoine arménien. Il est une réponse du politique en direction de la communauté arménienne de Valence. Sa vocation initiale correspond à une volonté de rappeler la présence arménienne à Valence et, implicitement, de reconnaitre publiquement au niveau de la ville le génocide arménien. La mise en scène de l'immigration dans cette structure culturelle municipale s'effectue en premier lieu dans l'exposition permanente : celle-ci, en s'appuyant sur des travaux d'historiens, des témoignages, archives, objets et photographies de Valentinois d'origine arménienne, présente "un parcours idéal" du rescapé arménien ayant survécu aux massacres, transitant par le Proche-Orient et Marseille avant d'élire domicile à Valence dans les années vingt. De plus, c'est une institution qui, dès son ouverture, se donnera comme mission, dans son espace d'exposition temporaire et dans son centre de documentation, d'interroger les migrations et les génocides contemporains dans le monde selon une approche historique. Depuis son ouverture, le Centre accueille et réalise des expositions sur les thématiques "génocide/ exil/ diaspora/ migration". La première, Notre histoire, notre ville, Valence - Regards de collégiens (mai-juin 2006), fut réalisée avec des élèves du collège Gaston-Bachelard ${ }^{(11)}$. Un an après, en partenariat avec la Cité nationale de l'histoire de l'immigration (Paris), le Centre du patrimoine arménien réalise l'exposition Se reconstruire en exil, l'arrivée des réfugiés arméniens en France (mars-avril 2007). Puis, en 2008, le lieu va exposer un travail photographique réalisé dans le sud de la France, "à la rencontre des saisonniers agricoles étrangers, [...] dans leur quotidien en marge de nos sociétés modernes ${ }^{(12) ": ~ L e s ~ D a m n e ́ s ~ d e ~ l a ~ s e r r e ~: ~ s a i s o n n i e r s ~ d a n s ~ l ' a g r i c u l t u r e . ~}$ Le lieu va confirmer sa volonté d'évoquer les migrations liées à des événements politiques et naturels. En 2009, il expose le travail photographique de Milomir Kovacevic, Sarajevo dans le cceur, où des Sarajéviens réfugiés à Paris présentent l'objet qu'ils ont emporté dans leur exil et le commentent. En 2010 et 2011, l'immigration va être évoquée avec le travail photographique du collectif Argos, Avec les réfugiés climatiques, parti à la rencontre de ceux qui sont déjà des réfugiés climatiques puis Interstices Urbains, une réflexion photographique et sonore sur les bidonvilles contemporains en région Rhône-Alpes ${ }^{(13)}$.

Un autre lieu culturel institutionnel ouvert à Villeurbanne (Rhône) depuis 2008 est propice à la muséographie de l'immigration. C'est le Rize/Centre mémoires et 
société. Le bâtiment regroupe les archives municipales, une bibliothèque de quartier, un espace d'exposition et une salle de spectacle/projection/conférence. Le Rize souhaite interroger notamment l'histoire, les mémoires, le patrimoine culturel immatériel de la ville. À ce titre cet établissement culturel a, à ce jour, réalisé deux expositions d'envergure qui interrogent l'immigration. La première, Olivier de Serre, radiographie d'une "cité ghetto"(2009), est un projet fortement historique : il s'appuie sur les archives d'un quartier aujourd'hui démoli et a fait appel à une historienne contemporaine de Lyon pour la réalisation des textes d'exposition. L'autre projet vient de s'achever : Musiques! Voyages sonores à Villeurbanne (2010). Il est l'aboutissement de deux années de collecte de musiques populaires - en particulier d'immigration - dans la ville, confié au Centre des musiques traditionnelles RhôneAlpes (CMTRA) par la municipalité de Villeurbanne. Pour cette exposition, l'immigration est mise en scène au travers d'un travail ethnologique et par la valorisation d'une collecte de sources orales.

\section{L'immigration, un objet muséographique large}

Cette série d'expériences muséographiques met en évidence l'intérêt progressif pour évoquer l'immigration dans les institutions culturelles en Rhône-Alpes. Cela va de pair avec la reconnaissance grandissante de l'immigration comme objet historique et le phénomène de mode qui entoure la notion de mémoire.

Aujourd'hui, ce phénomène est devenu, en grande partie, un objet légitime partagé par beaucoup de musées et d'institutions culturelles telles que les centres d'interprétation, les bibliothèques et les services d'archives. La création à Paris de la Cité nationale de l'histoire de l'immigration ouverte en 2007 est un exemple de réponse par le politique à cette dynamique qui traverse l'ensemble de l'Hexagone depuis les années quatre-vingt-dix.

En fonction des missions de chacune des institutions évoquées ci-dessus, on constate que la manière d'évoquer le sujet va être extrêmement hétérogène. Cela s'explique notamment par la reconnaissance de l'immigration comme phénomène qui traverse complètement notre société. En effet, la tendance générale montre aussi qu’à travers les expositions traitant d'immigration, il y a une volonté de questionner de plus en plus l'histoire d'un territoire tout en proposant des pistes de compréhension à travers une mise en tension de cette thématique dans une histoire et une actualité internationales. Ici un Musée de la résistance devient un espace pour évoquer les droits de l'homme, là un Centre du patrimoine arménien s'ouvre aux génocides et aux migrations de manière plus globale. 


\section{Notes}

1. "Traces en Rhône-Alpes" est le nom d'un événement artistique et culturel initié par l'association lyonnaise de bailleurs sociaux ARALIS. C'est un projet qui s'est appuyé au départ sur des acteurs institutionnels (le Fonds d'action social, la DRAC et la région) et des acteur associatifs installés à Grenoble, Saint-Étienne et Vaulx-en-Velin (Adate, la revue Écarts d'identité, Le Grain et Peuplement \& Migrations). À partir de 2005 et jusqu'à 2009, la biennale Traces a joué un rôle de catalyseur pour une diversité d'acteurs rhônalpins désireux d'organiser sur leur territoire des événements culturels destinés à valoriser l'histoire et les mémoires des immigrations en région Rhône-Alpes. 2. Jean-Claude Duclos, "L'immigration au cceur du Musée dauphinois", in Écarts d'identité, n 108 , Faire mémoire. Traces des migrants en Rhône-Alpes, Grenoble, Adate Éditions, juin 2006, pp. 16-26. http://www.revues-plurielles.org/_uploads/pdf/6_108_4.pdf.

3. ALIF (Amitié et liens France-Maghreb) est l'héritière de l'Association dauphinoise de solidarité franconord-africaine fondée en 1955 qui fédérait un très grand nombre de travailleurs immigrés.

4. Léla Bencharif et Virginie Milliot, Traces et mémoires des migrations dans la région Rhône-Alpes. Parcours avant l'Escale, Vaulx-en-Velin, DRAC Rhône-Alpes, "Peuplement et migrations", 1999.

5. Daniel Pelligra, "L'Escale, cité du voyage", in Hommes é Migrations, $n^{\circ}$ 1247, janvier- février 2004, pp. 85-90.

6. TASE : Textile artificiel du Sud-Est.

7. Cette exposition fut coréalisée avec Traces-Aralis et l'association le Grain, en partenariat avec deux autres institutions dépositiaires de fonds photographiques : le Musée dauphinois et les archives municipales de Saint-Étienne, respectivement en possession des fonds Honoré Parise et Léon Leponce .

8. Collecte et montage réalisés par Péroline Barbet/Centre des musiques traditionnelles Rhône-Alpes.

9. Vincent Lemire et Stéphanie Samson, Baraques, l'album photographique du dispensaire La Mouche-Gerland (1929-1936), Lyon, Le temps qu'il fait, ENS-Lyon, 2003.

10. Marie-Claude Blanc-Chaléard, "Des baraques aux bidonvilles", in Vincent Lemire et Stéphanie Samson, Baraques, op. cit., pp. 89-92.

11. Le collège se situe dans le quartier Fontbarlette de Valence, où une proportion importante des élèves sont immigrés ou enfants d'immigrés.

12. Brochure du Centre du patrimoine arménien présentant l'exposition photographique Les Damnés de la serre, de Yohanne Lamoulère, CPA Valence, 2008.

13. Interstices urbains. Photographies et texte: Benjamin Vanderlick. Création sonore : Jean-Baptiste Fribourg, $11 \mathrm{~min}$, Valence, 2011. 